Western University

Scholarship@Western

Brain and Mind Institute Researchers'

Publications

Brain and Mind Institute

8-1-2015

\title{
Transient visual responses reset the phase of low-frequency oscillations in the skeletomotor periphery.
}

\author{
Daniel K Wood \\ Brain and Mind Institute, University of Western Ontario, London, ON, Canada \& Department of \\ Neurobiology, Northwestern University, 2205 Tech Dr., Hogan 2-160, Evanston, IL, 60208, USA \\ Chao Gu \\ Brain and Mind Institute, University of Western Ontario, London, ON, Canada \& Graduate Program in \\ Neuroscience, University of Western Ontario, London, ON, Canada \& Robarts Research Institute, London, \\ ON, Canada \\ Brian D Corneil \\ Brain and Mind Institute, University of Western Ontario, London, ON, Canada \& Robarts Research Institute, \\ London, ON, Canada \& Departments of Psychology, Physiology and Pharmacology, University of Western \\ Ontario, London, ON, Canada \\ Paul L Gribble \\ Brain and Mind Institute, University of Western Ontario, London, ON, Canada \& Departments of \\ Psychology, Physiology and Pharmacology, University of Western Ontario, London, ON, Canada \\ Melvyn A Goodale \\ Brain and Mind Institute, University of Western Ontario, London, ON, Canada \& Departments of \\ Psychology, Physiology and Pharmacology, University of Western Ontario, London, ON, Canada \\ Follow this and additional works at: https://ir.lib.uwo.ca/brainpub \\ Part of the Neurosciences Commons, and the Psychology Commons
}

\section{Citation of this paper:}

Wood, Daniel K; Gu, Chao; Corneil, Brian D; Gribble, Paul L; and Goodale, Melvyn A, "Transient visual responses reset the phase of low-frequency oscillations in the skeletomotor periphery." (2015). Brain and Mind Institute Researchers' Publications. 186.

https://ir.lib.uwo.ca/brainpub/186 


\title{
Transient visual responses reset the phase of low-frequency oscillations in the skeletomotor periphery
}

\author{
Daniel K. Wood, ${ }^{1,2}$ Chao Gu, ${ }^{1,3,4}$ Brian D. Corneil, ${ }^{1,4,5}$ Paul L. Gribble ${ }^{1,5}$ and Melvyn A. Goodale ${ }^{1,5}$ \\ ${ }^{1}$ Brain and Mind Institute, University of Western Ontario, London, ON, Canada \\ ${ }^{2}$ Department of Neurobiology, Northwestern University, 2205 Tech Dr., Hogan 2-160, Evanston, IL 60208, USA \\ ${ }^{3}$ Graduate Program in Neuroscience, University of Western Ontario, London, ON, Canada \\ ${ }^{4}$ Robarts Research Institute, London, ON, Canada \\ ${ }^{5}$ Departments of Psychology, Physiology and Pharmacology, University of Western Ontario, London, ON, Canada
}

Keywords: EMG, feedforward sweep, human, orienting reflex, physiological tremor, rapid reaching

\begin{abstract}
We recorded muscle activity from an upper limb muscle while human subjects reached towards peripheral targets. We tested the hypothesis that the transient visual response sweeps not only through the central nervous system, but also through the peripheral nervous system. Like the transient visual response in the central nervous system, stimulus-locked muscle responses $(<100 \mathrm{~ms})$ were sensitive to stimulus contrast, and were temporally and spatially dissociable from voluntary orienting activity. Also, the arrival of visual responses reduced the variability of muscle activity by resetting the phase of ongoing low-frequency oscillations. This latter finding critically extends the emerging evidence that the feedforward visual sweep reduces neural variability via phase resetting. We conclude that, when sensory information is relevant to a particular effector, detailed information about the sensorimotor transformation, even from the earliest stages, is found in the peripheral nervous system.
\end{abstract}

\section{Introduction}

The onset of a visual stimulus results in a transient burst of neural activity that sweeps in a feedforward manner throughout visual circuits (Boehnke \& Munoz, 2008). This response carries information about the location, but not the identity or task relevance, of the stimulus (Lamme \& Roelfsema, 2000). Recurrent activity appears later, integrating the pure visual response with top-down modulation, and distinguishing targets from distractors until a unique motor response is selected (Corbetta \& Shulman, 2002). Generally, this process is described as playing out fully in the cortex until a decision is made and a movement is planned, after which the motor commands descend to the spinal cord. Here, we demonstrate that even the earliest stages of visuomotor processing are propagated to the skeletomotor periphery.

We tested whether the transient visual response is propagated to muscles in the human upper limb during a reaching task. This has the potential to explain a wide range of reflexive reaching phenomena, including the manual following response (Saijo et al., 2005), rapid online correction (Day \& Lyon, 2000), and the global effect in reaching (Wood et al., 2011). We recorded intramuscular activity from chest muscles while subjects reached to peripheral visual targets. Consistent with previous reports (Corneil et al., 2004; Pruszynski et al., 2010), we observed stimulus-locked responses (SLRs) in human upper limb muscles. These SLRs shared defining features of

Correspondence: Daniel K. Wood, ${ }^{2}$ Department of Neurobiology, as above.

E-mail: danielkentwood@gmail.com the transient visual response: they were sensitive to the contrast of the stimulus, they were temporally and spatially dissociable from voluntary orienting, and they were evoked by stimulus offsets (Gawne, 2002). Together, these findings suggest that the transient visual response sweeps not only through the central nervous system, but also through relevant segments of the peripheral nervous system.

Oscillatory dynamics in the electromyographic (EMG) recordings supported this hypothesis. As sensory information sweeps through the brain, it resets the phase of ongoing oscillations (Lakatos et al., 2008), thereby reducing neural variability (Churchland et al., 2010). Sensory-induced phase resetting selectively modulates the processing of later recurrent activity (Schroeder \& Lakatos, 2009), and the resulting drop in neural variability may serve as a threshold mechanism for movement initiation (Churchland et al., 2006; Purcell et al., 2012). Thus, sensory-induced phase resetting appears to play a role in the transition between sensory selection and the initiation of voluntary movement (Nicolelis et al., 1995). Here, the SLR selectively reset the phase of ongoing low-frequency oscillations, resulting in a reduction in the variability of muscle activity. Therefore, detailed information about the transformation of a sensory signal into a voluntary movement command is expressed in upper limb muscles prior to reaching.

\section{Materials and methods}

A total of 15 human subjects (aged 21-41 years; all male) participated with informed consent, and were paid for their participation. Six of the subjects participated in both of the experiments reported 
here. All procedures were approved by the University Research Ethics Board for Health Science Research at the University of Western Ontario. All subjects reported no history of visual, neurological or musculoskeletal disorder. One of the subjects was left-handed; all others were right-handed.

\section{Apparatus}

Subjects performed reaching movements while grasping the handle of a robotic manipulandum (InMotion Technologies) with their right hand (Fig. 1a). A six-axis force transducer (ATI Industrial Automation, Apex, NC, USA; resolution, $0.05 \mathrm{~N}$ ), which was located inside the handle, measured manual forces. The position of the manipulandum in the horizontal plane was sampled at $600 \mathrm{~Hz}$. Subjects sat at a desk and interacted with the robot in a horizontal plane at shoulder height. A custom air sled, secured below the subject's right elbow, supported the arm during movements. All stimuli were presented on a horizontal mirror (placed just below chin height) that reflected the display of a downward-facing LCD monitor. The mirror occluded the subject's view of the arm. Real-time visual feedback of hand position was provided by means of a small red dot projected onto the mirror by the LCD monitor. The precise timing of visual events on the screen was determined with a photodiode.

\section{Muscle electromyography}

We recorded EMG activity from the clavicular head of the right pectoralis major (cPM), a muscle involved in generating torque at the shoulder joint during whole-arm reaching movements. Recordings were made with intramuscular fine-wire electrodes, by the use of staggered monopolar insertions to characterise cPM recruitment across multiple motor units. Six insertions (i.e. three channels with two electrodes each) were spaced $\sim 1 \mathrm{~cm}$ apart, typically $1 \mathrm{~cm}$ below (inferior to) the clavicle, with the most lateral insertions being placed just under the lower clavicular convexity (Fig. 1b). A surface ground electrode was placed on the left clavicle. EMG data were recorded with a Myopac Jr system (Run Technologies; low-pass filter modified to $2 \mathrm{kHz}$ ). The EMG data were amplified and sampled at $4 \mathrm{kHz}$. Offline, EMG signals were then full-wave rectified and bin-integrated into 1-ms bins.

\section{Experimental tasks}

In both tasks, subjects were instructed to move as quickly as possible, and to overshoot the target. During piloting, we found that a higher baseline EMG signal, induced by a constant load force on the arm, had a beneficial effect on the detectability of the SLR. We therefore used the robotic arm to generate a constant load force of $5.3 \mathrm{~N}(5 \mathrm{~N}$ to the right; $1.75 \mathrm{~N}$ down) opposite to the direction of the upper left target from the starting position. For comparison, gravity exerts a constant force of $29.4 \mathrm{~N}$ on an outstretched arm with a mass of $3 \mathrm{~kg}$.

\section{Luminance contrast}

Subjects $(n=11)$ performed a center-out reaching task towards a single target. A trial started when the subject brought the cursor (a red dot representing real-time hand position) into a central start-position circle and maintained that position for $2.5 \mathrm{~s}$. The start-position circle then changed color to signal the beginning of the trial. After 1-1.5 s (randomised), the start-position circle disappeared. Exactly $200 \mathrm{~ms}$ later, a target appeared $10 \mathrm{~cm}$ from the start-position point, in one of two locations: (i) $160^{\circ}$ (i.e. upper left target) from the start-position point; or (ii) $340^{\circ}$ (i.e. lower right target) from the start position. The 200-ms 'gap' was used because of its role in hastening arm movements (Gribble et al., 2002) and potentiating the visual grasp reflex (Fischer \& Boch, 1983), and the possibility that these effects could lead to a more reliable elicitation of the SLR.
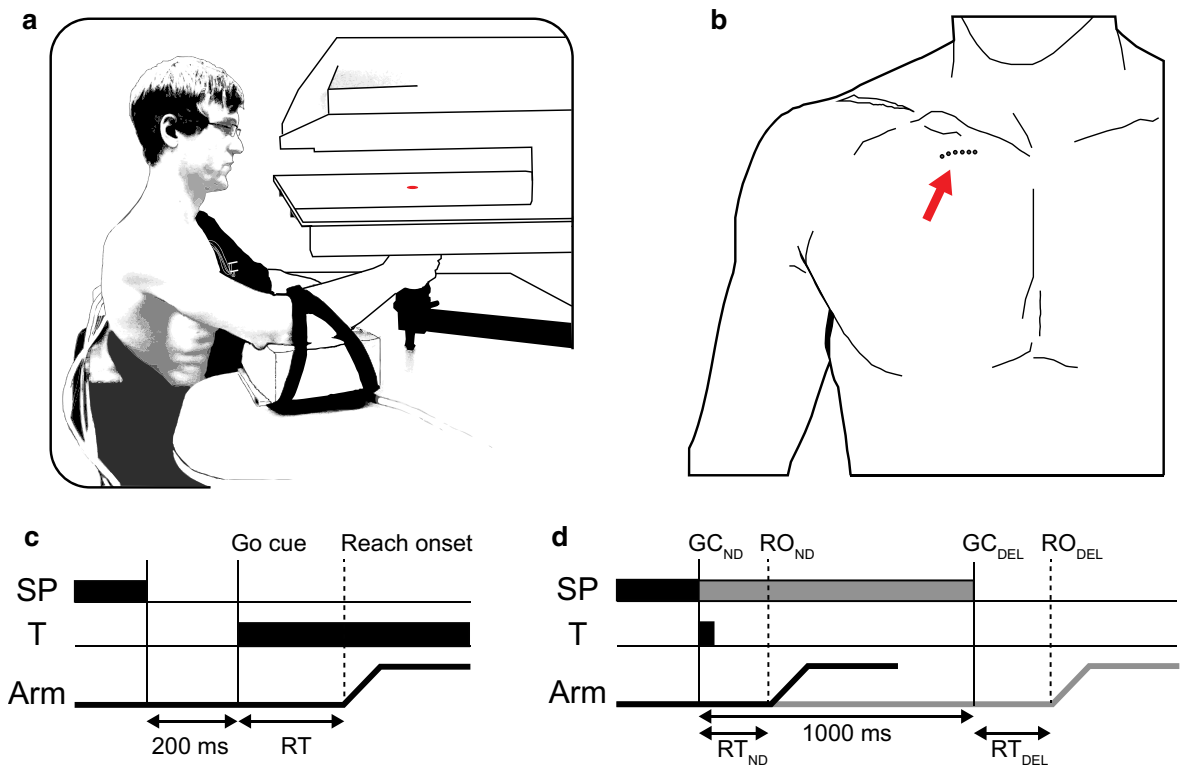

FIG. 1. Experimental paradigm. (a) Subjects held the handle of a robotic arm, with their arm supported by an air sled. They viewed both the reach targets and a cursor representing the real-time position of their hand on a mirror surface that reflected the output of a downward-facing LCD screen. (b) The arrow indicates the approximate placement of electrodes in the cPM. (c) Trials in the luminance contrast task started with subjects holding the cursor in the central start-position circle (SP). The SP disappeared $200 \mathrm{~ms}$ prior to target (T) onset, after which subjects immediately reached towards the target. (d) Trials in the delay task also started with the cursor in the SP. A peripheral target then briefly flashed for $150 \mathrm{~ms}$. The SP disappeared either at target onset [black bar; GC $\mathrm{ND}\left(\mathrm{Go}_{\mathrm{C}} \mathrm{cue}\right.$, No Delay)], or after a 1-s delay [gray bar; Go-cue, Delay $\left(\mathrm{GC}_{\mathrm{DEL}}\right)$ ]. SP disappearance was the cue to initiate the reach [reach onset $\left.(\mathrm{RO})\right]$ towards the location of the flashed target. 
We used four different levels of target luminance contrast. The targets were circles filled with different shades of gray against a white background $\left(383.3 \mathrm{~cd} / \mathrm{m}^{2}\right)$. The lowest-contrast target $\left(370.5 \mathrm{~cd} / \mathrm{m}^{2}\right)$ had a Weber contrast ratio of $3.3 \%$. The second lowest-contrast target $\left(352.4 \mathrm{~cd} / \mathrm{m}^{2}\right)$ had a Weber contrast ratio of $8.1 \%$. The second highest-contrast target $\left(311 \mathrm{~cd} / \mathrm{m}^{2}\right)$ had a Weber contrast ratio of $18.9 \%$. Finally, the highest-contrast target $\left(0.4 \mathrm{~cd} / \mathrm{m}^{2}\right)$ had a Weber contrast ratio of $99.9 \%$. Luminance was measured with a Konica Minolta CA-100 photometer.

\section{Delayed reaching}

Subjects $(n=10)$ performed an immediate/delayed reaching task. After a variable inter-trial interval, subjects were presented with a single target in one of the two locations described in the luminance contrast task. Targets had a Weber contrast ratio of $99.9 \%$. Subjects were instructed to initiate the reach only after the disappearance of the central start-position circle. This could happen either: (i) immediately, concurrent with target onset; or (ii) $1000 \mathrm{~ms}$ after target onset. Critically, the no delay and delay trials were randomly interleaved. In both conditions, the target was on the screen for only $150 \mathrm{~ms}$. The target reappeared momentarily once the hand reached it.

\section{Data analysis}

\section{Kinematic analysis}

In order to achieve sample-to-sample locking between kinematic and EMG data, kinematic data were up-sampled from $600 \mathrm{~Hz}$ to $1000 \mathrm{~Hz}$ with a lowpass interpolation algorithm, and then lowpassfiltered with a second-order Butterworth filter, with a cutoff at $150 \mathrm{~Hz}$. Reaction time (RT) was calculated as the time from the appearance of the reach target, as measured by the photodiode located on the LCD screen (luminance contrast task), or the disappearance of the central start-position point (delay task) to the initiation of the reach. Reach initiation was identified by first finding the peak tangential hand velocity, and then moving backwards to the closest previous point at which the velocity profile reached $5 \%$ of the peak. Errors in reach direction were determined by: (i) detecting suprathreshold velocity bumps prior to RT definition; and/or (ii) sampling the position of the hand $100 \mathrm{~ms}$ after reach initiation. If the position was not within $\pm 45^{\circ}$ of the true target location, the reach was classified as an error and was excluded from analysis. As the RT distributions with the longest tails extended into the 600-ms range, RTs slower than $700 \mathrm{~ms}$ were also excluded from analysis.

\section{Receiver-operating characteristic (ROC) analysis}

ROC analysis was used to determine the presence and timing of stimulus-locked activity in the cPM muscle recordings (Corneil et al., 2004; Pruszynski et al., 2010). We separated EMG waveforms by target location and target contrast (luminance contrast task) or delay duration (delay task). These waveforms were then smoothed with a seven-point $(7 \mathrm{~ms})$ running average. For every sample $(1 \mathrm{~ms})$ between $100 \mathrm{~ms}$ before and $300 \mathrm{~ms}$ after target presentation (both tasks) or start-position disappearance (delay task), we calculated the area under an ROC curve. This metric indicated the probability that an ideal observer could discriminate between a leftward (cPM as agonist) and rightward (cPM as antagonist) reaching movement, based on the distribution of EMG activity at that particular sample. A value of 0.5 indicates chance discrimination, whereas values of 0 and 1 indicate perfect discrimination. We set the threshold for discrimination at 0.675 (or 0.325 for the opposite direction), which is similar to (although slightly more conservative than) what was used in Pruszynski et al. (2010). The time of earliest discrimination was defined as the time after stimulus onset (luminance contrast task) or start-position disappearance (delay task) at which the ROC area surpassed 0.675 , and remained above that threshold for at least five of the next 10 samples.

One of the primary goals of the present study was to test whether or not the luminance contrast of a target modulates the timing of stimulus-locked muscle activity. We used the ROC analysis to address this question. There were two practical hurdles, however. First, differences between target contrast conditions in the distribution of RTs meant that any differences in the timing of the earliest ROC discrimination for a given condition might simply be a function of the underlying RT distribution. The second practical hurdle was that RTs were often very fast, even in conditions where no directional errors were committed (that is, subjects were not merely guessing). Often, the large burst of muscle activity associated with the voluntary movement for these earliest RTs overlapped considerably with the temporal range in which stimulus-locked activity occurs. This had the effect of washing out any potential signature of the stimulus-locked activity.

We addressed these difficulties by removing trials with the earliest RTs (and, by extension, EMG activity that overlapped with the range of interest), thus simplifying the process of distinguishing between the presence and absence of stimulus-locked activity. Specifically, we removed trials in ascending order of RT while dividing the remaining trials into 'early' and 'late' RT groups (Fig. 3a) and finding the slope of the relationship between the average RT and the earliest discrimination times for these two groups (Fig. 3b and c). Whenever the slope of this relationship exceeded $67.5^{\circ}$ (i.e. halfway between unity at $45^{\circ}$ and vertical at $90^{\circ}$ ), we combined the RT groups and performed the ROC analysis again. Our goal was to identify an actual peak in the combined ROC function near the discrimination times for the separate ROC functions. This was done to avoid situations in which one RT group exceeded threshold because of movement-related activity rather than SLR-related activity. To find the peak, we first identified the point in the combined ROC function where three of 10 consecutive frames were above the discrimination threshold. We then defined a window from $10 \mathrm{~ms}$ before to $30 \mathrm{~ms}$ after this point, and within that window identified where the (135-Hz lowpassed) first derivative of the ROC function dipped below zero. If this peak detection algorithm could detect a peak in the ROC time course (Figs $3 b$ and $4 b$ ), we assumed the presence of stimulus-locked activity. If (i) the slope of the discrimination time and RT relationship between the groups failed to exceed $67.5^{\circ}$, or (ii) a peak was never detected in the combined ROC where the slope did exceed $67.5^{\circ}$, we assumed the absence of stimuluslocked activity.

Note that, in order for the analysis to reach the stage where the peak was detected, there had to be prior evidence that the timing of such a peak would be invariant with respect to RT. Thus, the latency of the ROC peak was assumed to be a faithful measure of the latency of stimulus-locked activity, in spite of differences between the RT distributions between target contrast conditions. We quantified the magnitude of the SLR for a given subject and contrast condition by taking the associated latency of the ROC peak and averaging the EMG activity within an 11-ms window with the peak latency at the center (i.e. $5 \mathrm{~ms}$ before to $5 \mathrm{~ms}$ after the peak). To normalise between electrodes (given the large variability in mean EMG signal strength between electrodes), we then subtracted a baseline EMG value, calculated by taking the average of EMG activity 
$100 \mathrm{~ms}$ prior to stimulus presentation. As we were interested in the within-subject relationships between SLR magnitudes for the various contrast conditions, and not patterns of SLR magnitude between subjects, we normalised the magnitude measures within subjects. Accordingly, we divided the magnitude score for each contrast condition by the within-subject mean score across all significant contrast conditions. One subject ( $\mathrm{s} 9$ from Fig. $2 \mathrm{f}$ ) had only one significant SLR condition, and was therefore excluded from the magnitude analysis. We performed ANOvas and planned comparisons (using
Welch's $t$-test, owing to unequal sample sizes) on the peak latencies and average SLR magnitudes for the four target contrast conditions. The $P$-values for reported $t$-tests are therefore uncorrected.

\section{Modified ROC analysis for the delay experiment}

The approach to characterising the SLR in the luminance contrast experiment emphasised the invariance of ROC detection time with respect to RT. Specifically, two separate ROC analyses were performed on early
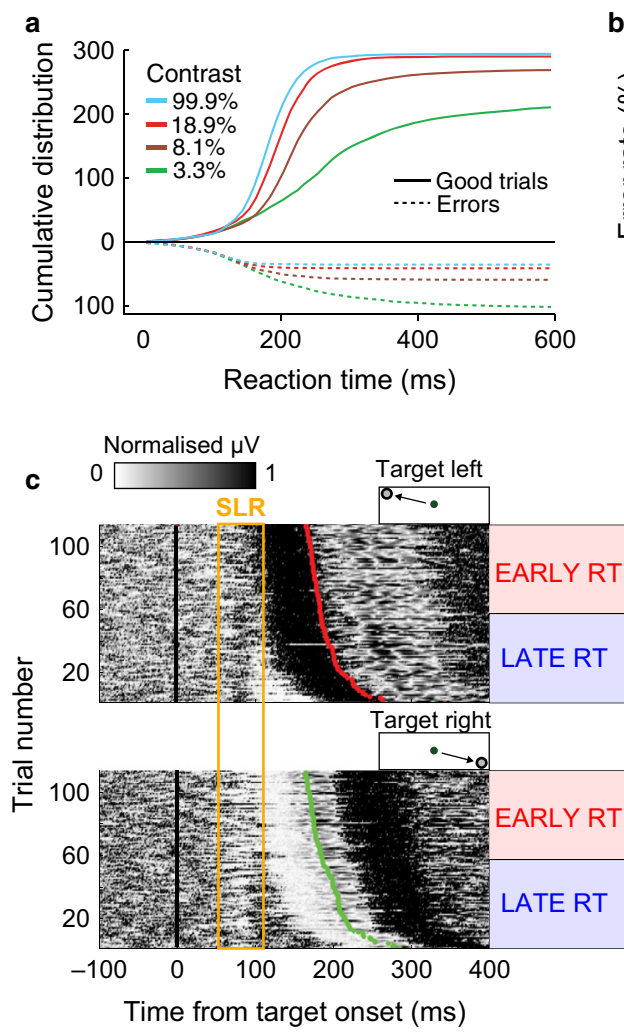
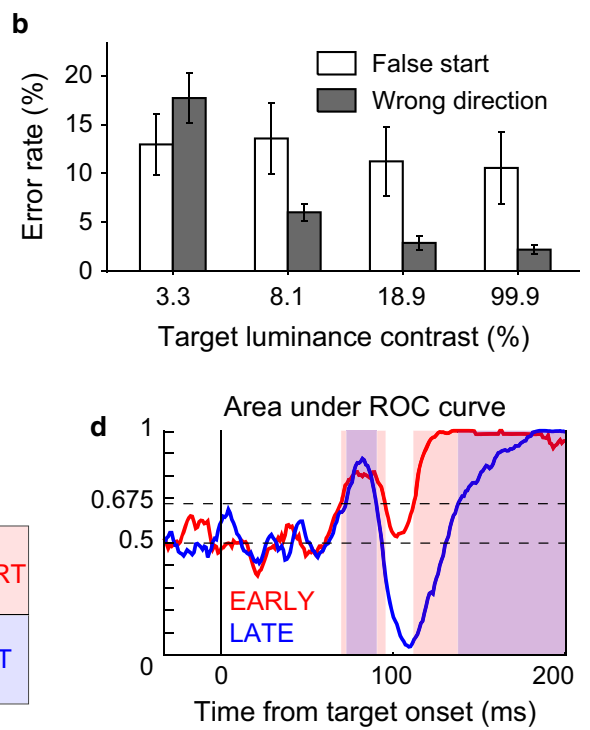

e

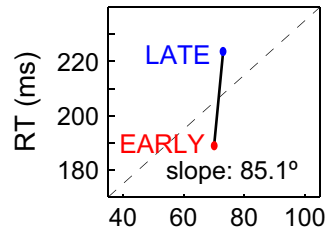

Discrimination time $(\mathrm{ms})$

Luminance contrast

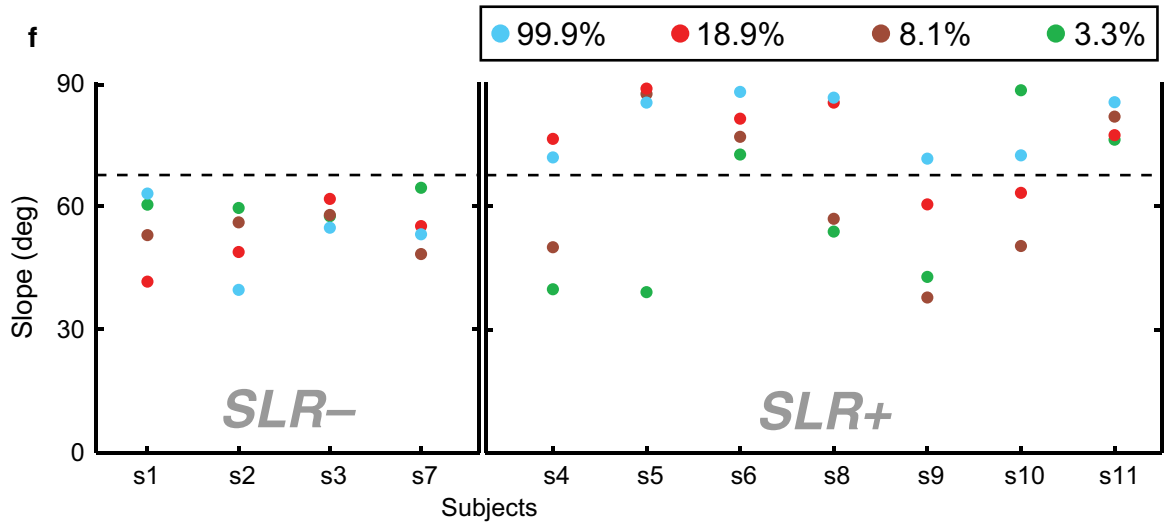

FIG. 2. Behavioral performance and detection of stimulus-locked activity. (a) Cumulative RT distributions for the four contrast conditions. (b) Between-subject mean error rates. Error bars are SEMs. (c) EMG magnitude over time for Target Left (top panel) and Target Right (bottom panel) trials. Trials are sorted by RT and locked to visual target onset (black vertical line at time 0). Darker colors represent greater EMG activity, normalised to peak activity for display purposes (hence the lack of scale). Manual RT for each trial is marked with red (Target Left) or green (Target Right) circles. In the intensity plots, the SLR appears as a vertical band (see orange box) of excitation (Target Left) or suppression (Target Right) locked to stimulus onset, regardless of RT. Trials were separated into early (red) or late (blue) RT groups. (d) Area under the ROC curve was calculated for both RT groups. ROC discrimination time (RDT) occurred when the ROC area first surpassed a value of 0.675. Red (early RT) and blue (late RT) bands indicate samples at which the corresponding ROC area is over threshold. (e) The slope of the relationship between RDT and average RT for early and late RT groups was calculated. (f) Each data point represents, for a given subject, the slope of the relationship between RDT and RT for the fast and slow RT trials, and the color of the dot corresponds to different target contrast conditions (see legend). The subjects are grouped into those who showed an SLR in at least one target contrast condition (SLR+) and those who did not (SLR-), given a slope threshold of $67.5^{\circ}$ (dotted line). 

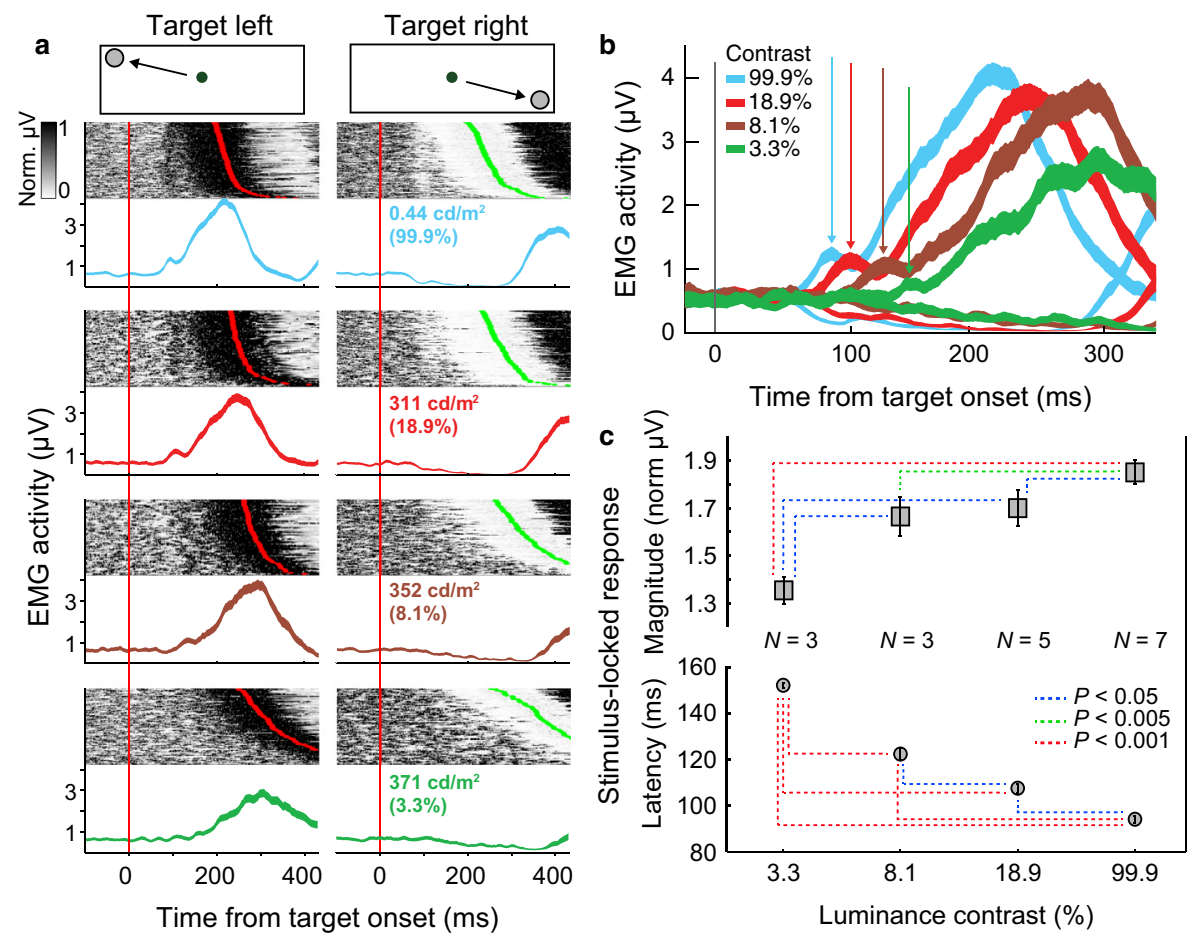

FIG. 3. The effect of luminance contrast on the SLR. (a) Exemplar EMG recordings from the right cPM of a single subject in the luminance contrast experiment. Left column: Target Left trials (cPM acting as agonist). Right column: Target Right trials (cPM acting as antagonist). Rows correspond to different target contrast conditions. Colored text indicates the luminance of the target and the Weber contrast of target against background luminance. Data are aligned to visual target presentation (red vertical line at $0 \mathrm{~ms}$ ). For display purposes, grayscale intensity values are normalised to peak voltage. Mean EMG traces are shown below each intensity subplot. The width of the trace subtends the SEM. (b) Mean EMG traces from a, overlaid to illustrate latency and magnitude differences across contrast conditions. Arrows point to SLR activity. (c) Mean latency and magnitude values for performances where an SLR was detected, plotted for each contrast condition. Colored dotted lines indicate results of $t$-tests. Error bars indicate SEMs.

and late RT trials, and the ROC discrimination times were regressed against average RT for the two RT bins. This approach was problematic for the delay experiment, for a number of reasons. First, in the No Delay condition, RTs were exceedingly long, owing to uncertainty introduced by the interleaving of 'immediate' and 'delay' trials. This uncovered the robust oscillation of the SLR, which was problematic, because the ROC discrimination could cross threshold at the first, second and, sometimes, the third cycle of the oscillation. This problematised attempts to relate ROC discrimination time to RT. Second, in the Delay Stim-locked epoch, there was no RT. Third, in the Delay Go-locked epoch, the Target Left and Target Right SLRs oscillated synchronously. As the ROC analysis was an attempt to discriminate reach direction on the basis of differences between Target Left and Target Right EMG activity, the inphase oscillations were problematic.

To be consistent in analysis across the three epochs, we settled on a technique that does not regress early and late RT with discrimination times, and that does not rely on running the ROC with Target Left vs. Target Right. To justify not using the RT bins, we point to previous reports (Corneil et al., 2004; Pruszynski et al., 2010), and the infrequency of having the regression of RT with discrimination times of early, non-voluntary activity fail to meet the slope threshold for SLR significance in our own dataset. At this point, we consider it a well-established empirical finding that when there is SLR activity, it remains temporally invariant with respect to RT.

Our modified approach involved running the ROC analysis separately for Target Left and Target Right EMG data. The EMG data were evaluated against a noise vector with the same mean and standard deviation as the baseline activity for the recording being analysed. This was done for each time point in the recording. The resulting ROC function was lowpass-filtered (50-Hz cutoff) to pre- vent spurious spikes from passing threshold, which was set to \pm 3 standard deviations of the first $150 \mathrm{~ms}$ of the ROC function. We then identified the peak in the ROC function corresponding to the voluntary activity. If both the Target Left and the Target Right ROC functions surpassed threshold prior to the voluntary activity, the SLR was scored positively. If only one of these passed threshold, it was scored negatively, to be conservative.

\section{Evaluating SLR spatiotemporal features}

To characterise the temporal relationships between Target Left and Target Right SLR activity in the various epochs of the delay experiment, we treated the SLRs effectively as sine waves, and used the phase as a proxy for the relative timing of the SLR peaks. We took the within-subject average EMG trace around the SLR (i.e. a window starting at $50 \mathrm{~ms}$ and ending just before the voluntary activity, which was typically between 150 and $250 \mathrm{~ms}$ ) for a given condition, and found the Fourier basis function within the $10-15-\mathrm{Hz}$ band that gave the best fit for both movement directions. We then calculated the difference in phase between the two fits. We used this as an indirect measure of reciprocal contraction of agonist and antagonist muscles (inferred from the relationship between Target Left and Target Right fits), which would correspond to $\pi$ radians of phase difference in the limit, or co-contraction, which would correspond to a lack of phase difference between agonist and antagonist.

\section{Spectral analysis}

In Fig. 4c-e, we show examples of emergent $10-15-\mathrm{Hz}$ oscillations in mean EMG traces at the time of the SLR. For all Fourier 

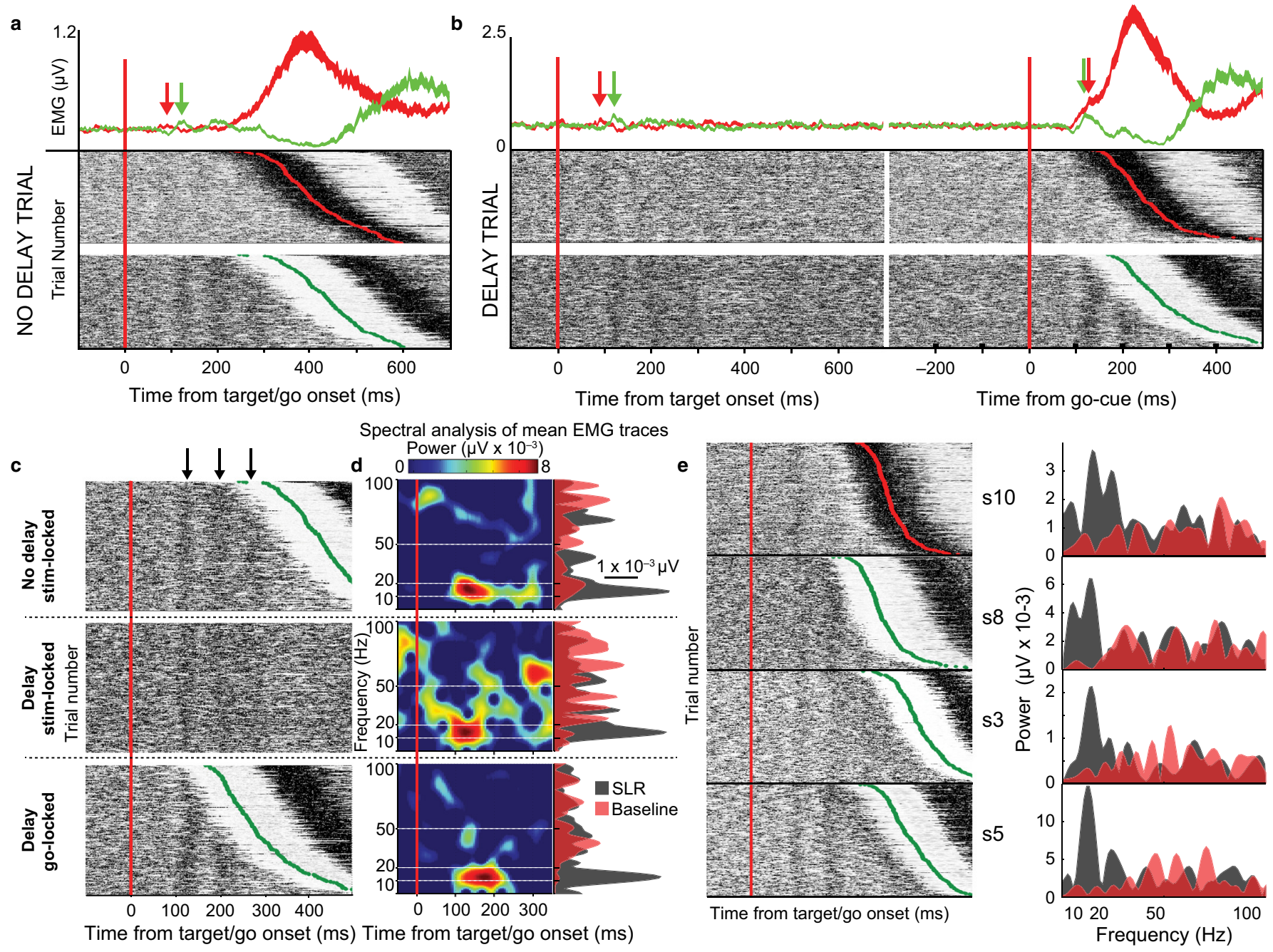

FIG. 4. Oscillatory SLRs in the instructed delay experiment. (a and b) Intensity supblots represent EMG activity for individual trials, sorted by RT (red dots for left target RT; green for right target RT). Mean EMG traces are plotted above. Red arrows indicate times of SLR for Target Left movements. Green arrows indicate rebound of Target Right SLR. For display purposes, grayscale intensity values are normalised to peak voltage. (a) No Delay condition, locked to target/ go-cue onset. (b) Delay condition, locked to target onset (first half) and go-cue (second half). Approximately 150 trials are depicted on the ordinate of each plot. (c) EMG activity (single subject) for all three epochs, sorted by RT and locked to target onset or go-cue. Black arrows indicate positive phases of 10-15-Hz oscillations. (d) Spectrograms of mean EMG waveforms, corresponding to the epochs in c. Horizontal white lines are 10, 20, and 50 Hz. Single-sided power spectra of mean EMG waveforms are plotted alongside the spectrograms. We compared spectral power from two 150-ms windows-of-interest: the SLR window is $75-225 \mathrm{~ms}$ post-stimulus, and the baseline window is $100 \mathrm{~ms}$ pre-stimulus to $50 \mathrm{~ms}$ post-stimulus. (e) Demonstration of SLR oscillation in raw EMG and power spectra plots for four other subjects, with the same conventions as in $\mathrm{d}$.

transforms, FFT length was set to the number of samples and NFFT to the next power of 2 above FFT length. For the time-frequency plots, the window size was 100 , the overlap was 90 , and each segment was windowed with a Hamming window. In Fig. 4, the power spectra are evaluated on the mean EMG trace. In Fig. 6, the power spectra are evaluated on a given epoch, with all trials appended together in a single vector.

\section{Phase resetting analysis}

To characterise phase resetting in the EMG data, we used EEGLAB (Delorme \& Makeig, 2004) and custom MATLAB scripts to calculate event-related spectral perturbation (ERSP) and inter-trial phase coherence (ITPC). As ERSP is a baseline-normalised spectrogram, it indicates spectral perturbations introduced by a time-locked experimental event. For $n$ trials, if $F_{k}(f, t)$ represents the spectral estimate of the $k$ th trial at frequency $f$ and time $t$, the ERSP is computed as:

$$
\operatorname{ERSP}(f, t)=\frac{1}{n} \sum_{k-1}^{n}\left|F_{k}(f, t)\right|^{2}
$$

where $\mid \mathbf{O}$ represents the complex norm. $F_{k}(f, t)$ was computed by use of a Morlet wavelet transform. Window size decreased and number of cycles increased linearly with frequency, from three cycles in a $389-\mathrm{ms}$ window at the lowest frequency $(8 \mathrm{~Hz})$ to 10.5 cycles in a $175-\mathrm{ms}$ window at the highest frequency $(60 \mathrm{~Hz})$.

The ITPC measure indicates the degree of inter-trial phase synchronisation at frequency $f$ and time $t$. This measure has been referred to as the 'phase locking factor' (Tallon-Baudry et al., 1996), and is computed as (using the same conventions as in Eqn 1):

$$
\operatorname{ITPC}(f, t)=\frac{1}{n} \sum_{k-1}^{n} \frac{F_{k}(f, t)}{\left|F_{k}(f, t)\right|}
$$


The ITPC measure can take values between 0 (absence of phase synchronisation) and 1 (perfect phase synchronisation). Statistical significance was assessed by bootstrapping confidence intervals for median spectral estimates within SLR (100-150 ms) and baseline (100-50 ms pre-stimulus) windows.

\section{Results}

\section{Luminance contrast determines the latency and magnitude of SLRs}

Subjects reached towards a single target possessing one of four levels of target luminance, selected to span the full range of visual con- trast (Fig. 1c). Center-out reaching movements were directed to one of two target locations: upper left and lower right (Fig. 5c), corresponding to a voluntary contraction and relaxation, respectively, of the cPM. When target contrast was higher, RT was shorter (ANOVA: $P<10^{-17}$ ) (Fig. 2a). Increasing target contrast had no effect on false starts, but led to fewer directional errors $\left(P<10^{-8}\right)$ (Fig. 2b).

The SLR appeared (in the intensity plots such as those in Fig. 2c) as a vertical band of increased contraction or relaxation preceding the voluntary movement-related muscle activity. We used ROC analysis to determine the presence, latency and magnitude of the SLR in each subject. Trials were equally divided into 'early' and 'late' RT bins (Fig. 2c). A separate ROC analysis was performed on the two different RT bins (Fig. 2d). When the slope of the relation-

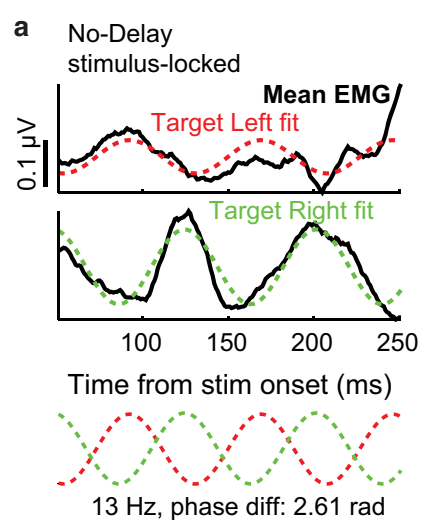

b

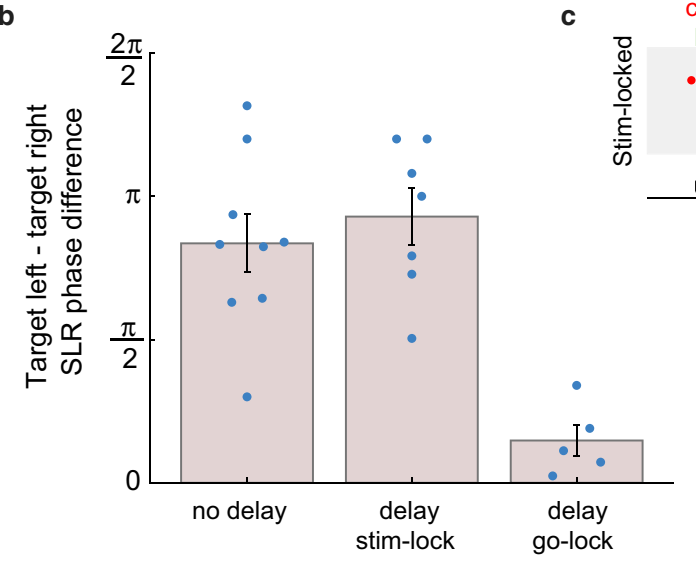

d

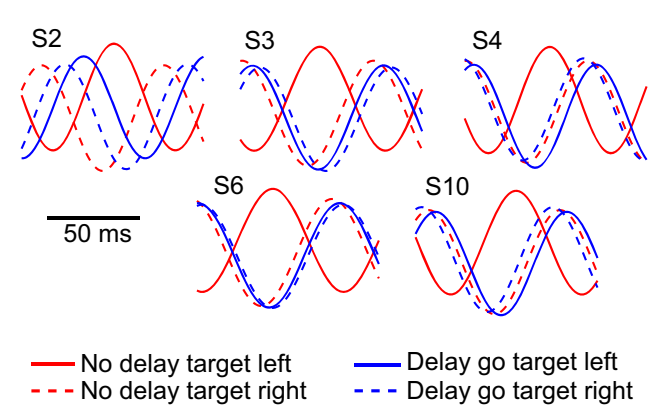
- - - No delay target right - - - Delay go target right

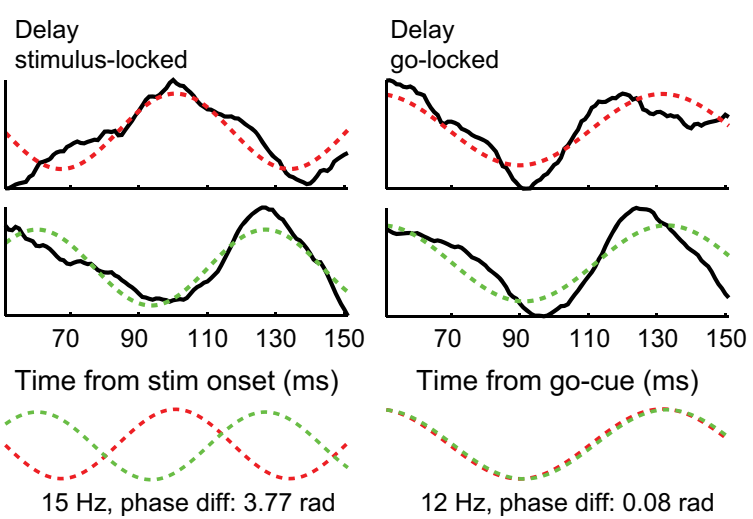

$12 \mathrm{~Hz}$, phase diff: $0.08 \mathrm{rad}$
cPM contraction $\quad$ cPM relaxation $\mathrm{pD}$ relaxation $\mathrm{pD}$ contraction
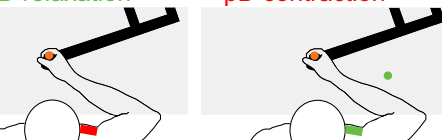
$\pi$ phase diff $\mathrm{NOOOC} \rightarrow$ Reciprocal contraction
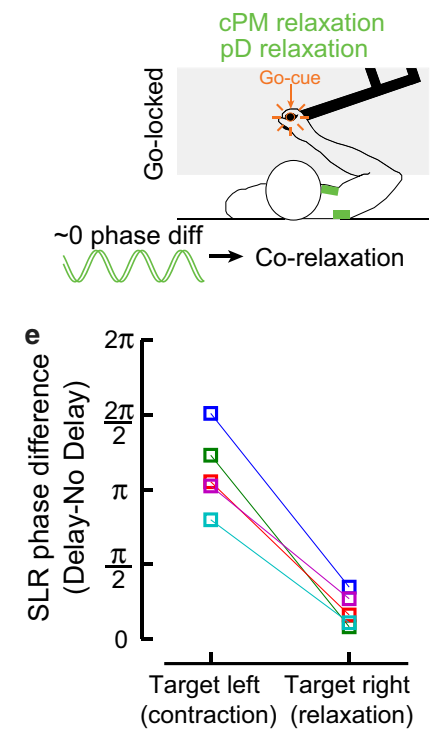

FIG. 5. Spatiotemporal relationships between SLRs. (a) Fourier fits to mean EMG trace (single subject shown here) at the time of SLR revealed reciprocal timing (anti-phase) between Target Left (top row) and Target Right (bottom row) SLRs for both Stim-locked epochs, and synchronous timing (in-phase) for the Delay Go-locked epoch. (b) Group-level phase differences between Target Left and Target Right SLR fits, for the three epochs. Blue circles are individual subjects. Error bars are between-subject SEMs. (c) Hypothesised agonist-antagonist relationships. Anti-phase fits suggest peripheral tuning (reciprocal contraction of agonist and antagonist muscles), whereas in-phase fits suggest a holding response (co-relaxation or co-contraction). pD, posterior deltoid. (d) For the five subjects with significant SLRs in both the No Delay and Delay Go-locked epochs, the No Delay Target Left fit is out of phase with the other fits. (e) The synchronous Delay Golocked SLRs are attributable to a delayed Target Left SLR (relative to No Delay SLRs for Target Left movements). Colors represent different subjects. 
ship between average RT and the earliest ROC discrimination time for the early and late trials exceeded a predetermined threshold $\left(67.5^{\circ}\right)$, we took it as evidence that activity was locked to the stimulus rather than to voluntary RT (Fig. 2e). Of the 11 subjects who participated in the luminance contrast experiment, seven showed an SLR in at least one of the contrast conditions (Fig. 2f). In a separate analysis, we regressed moving-window averages of ROC discrimination time and RT. The results of this supplemental analysis fully agreed with the results presented here (Fig. S1). Anecdotally, the ability to detect the SLR was correlated with the subject's body type; subjects with low muscle mass and low body fat most reliably produced recordings with an SLR. Given that all insertion needles were of identical length, penetrations went deeper into the muscles of thinner subjects. As previously suggested (Pruszynski et al., 2010), it is possible that the SLR is preferentially propagated to deeper muscle tissue (i.e. closer to the blood supply), where there is a higher concentration of slow-twitch fibers (Singh et al., 2002). Slow-twitch fibers are recruited earlier than fast-twitch fibers (Henneman, 1957), and are therefore more likely to mediate the lowlatency activity of the SLR.

We then compared the latencies of the first peaks past threshold in the ROC time course for each contrast condition. SLRs appeared sooner when target contrast was higher $\left(F_{3,14}=117.2, P<10^{-9}\right.$ (Fig. 3). Whenever SLRs were detected, we defined a $10-\mathrm{ms}$ window (i.e. $5 \mathrm{~ms}$ before and after the ROC peak) within which we integrated EMG activity, and then compared across target contrast conditions in order to test for differences in magnitude. SLRs had a larger magnitude when the target contrast was higher $\left(F_{3,13}=14.18\right.$, $P<0.001$ ) (Fig. 3).

\section{SLRs are spatially and temporally dissociated from voluntary orienting}

In a separate experiment, subjects performed an instructed delay task in which they reached towards the location of a briefly flashed (duration, $150 \mathrm{~ms}$ ) high-contrast target that appeared in one of the same locations as in the luminance contrast task (i.e. upper left or lower right). The imperative stimulus was the disappearance of the central start-position circle. This go-cue was given at one of two times: (i) no delay, concurrent with stimulus presentation; or (ii) delayed $1000 \mathrm{~ms}$ after stimulus presentation (Figs $1 \mathrm{~d}$ and $4 \mathrm{a}$ and b). Critically, these two conditions were randomly interleaved. Note that, except in rare instances of abnormally fast RTs in the No Delay condition, the target had already disappeared by the time when subjects started reaching towards it. RTs and error rates for this experiment are shown in Fig. S2.

Using a modified ROC analysis, we characterised SLRs in three separate EMG epochs: (i) No Delay; (ii) Delay Stim-locked (i.e. onset of target); and (iii) Delay Go-locked (i.e. disappearance of the start-position circle). The most consistent SLR was observed in the No Delay condition, with nine of 10 subjects showing an SLR. In the Delay conditions, seven of 10 subjects showed an SLR locked to the target onset, and five of 10 subjects showed an SLR locked to the go-cue (i.e. the disappearance of the central start-position circle). In summary, we found that the SLR appeared whenever the subject treated a visual event as an imperative stimulus, regardless of whether that visual event was a spatially informative target onset or a spatially uninformative offset of a central start-position marker. Furthermore, these SLRs appeared even when voluntary movements were suppressed.

During initial viewing of the raw data from this task, we noticed that the SLRs in the Delay Go-locked epoch were temporally syn- chronous rather than reciprocal between the Target Left and Target Right movements. To statistically validate this observation, we took advantage of the fact that the SLR oscillated within the $10-15-\mathrm{Hz}$ range (Fig. 4c-e), allowing us to treat the SLRs effectively as sine waves. We took the within-subject average EMG trace around the SLR for a given condition, and found the Fourier basis function within the $10-15-\mathrm{Hz}$ band that gave the best fit for both the Target Left and Target Right movements. We then calculated the difference in phase between the Target Left and Target Left fits (Fig. 5a). We used this as a measure of reciprocal contraction between agonist and antagonist muscles (inferred from the relationship between Target Left and Target Right fits), which would correspond to $\pi$ radians of phase difference in the limit, or co-contraction of the muscle pair, which would correspond to a lack of phase difference between Target Left and Target Right fits (Fig. 5c).

There was a significant effect of delay condition (i.e. No Delay; Delay Stim-locked; Delay Go-locked) on phase difference $\left(F_{2,18}=15.25, P=10^{-4}, \eta^{2}=0.63\right.$ (Fig. 5b). Phase differences between Target Left and Target Right fits for the Delay Go-locked condition $[M=0.46 \mathrm{rad}$, standard error of the mean $(\mathrm{SEM})=0.17]$ were different from those in the No Delay condition $(M=2.63 \mathrm{rad}$, SEM $\left.=0.32, t_{12}=4.78, P<0.0005\right)$ and the Delay Stim-locked condition $\left(M=2.92 \mathrm{rad}, \mathrm{SEM}=0.31, t_{10}=6.12, P<0.0005\right)$. The phase difference in the No Delay and Delay Stim-locked conditions did not differ $(P=0.53)$. In other words, SLRs in the No Delay and Delay Stim-locked conditions were characterised by reciprocal contraction (that is, phase differences between Target Left and Target Right fits were closer to $\pi$ than to 0), whereas SLRs in the Delay Go-locked condition were characterised by synchronous contraction (that is, phase differences were closer to 0 than to $\pi$ ). The synchronous contraction observed in the Delay Go-locked epoch was attributable to an initial relaxation rather than contraction (i.e. phase reversal) in the Target Left SLR (Fig. 5d and e), which indicates that the SLR for this epoch was an initial co-relaxation followed by oscillations of co-contraction and co-relaxation.

\section{Behavioral correlates of SLRS}

Kinematic evidence of the SLR was assessed with spatial position and force measurements from the manipulandum. We found no evidence of the SLR in the spatial position of the hand. This was probably attributable to: (i) the degrees of freedom intervening between the chest muscles and the hand; and (ii) the inertia of the robot arm, which should dampen small fluctuations in movement. However, there was evidence of the SLR in the force measurements. We calculated force rates (i.e. first derivative of force) for $x$-forces and $y$-forces during stimulus-locked epochs. To avoid overlap between voluntary forces and SLR-related forces, we used an RT cutoff (i.e. including only trials with manual RT of $>270 \mathrm{~ms}$ ) for the No Delay trials (as Delay trials had no RT in the stimulus-locked epoch). Target Left and Target Right $x$-force rates were significantly different for both No Delay $(P=0.0187)$ and Delay $(P=0.0019)$ trials during a 150-200-ms post-stimulus window, but not during a baseline (15-65 ms post-stimulus) window ( $P>0.5$ (Fig. 6). The same was true for $y$-force rates; No Delay $(P=0.0019)$ and Delay $(P=0.0448)$ trials were different during the 150-200-ms window, but not during the baseline window $(P>0.3)$.

There were two unexpected features of this bump in the force rates: it was in the opposite direction of the voluntary force rate profiles, and, at $150 \mathrm{~ms}$, it was later than the first wave of the SLR in the EMG recordings. Also, because this bump occurred just prior to the onset of force rates pertaining to the voluntary reach, we investi- 

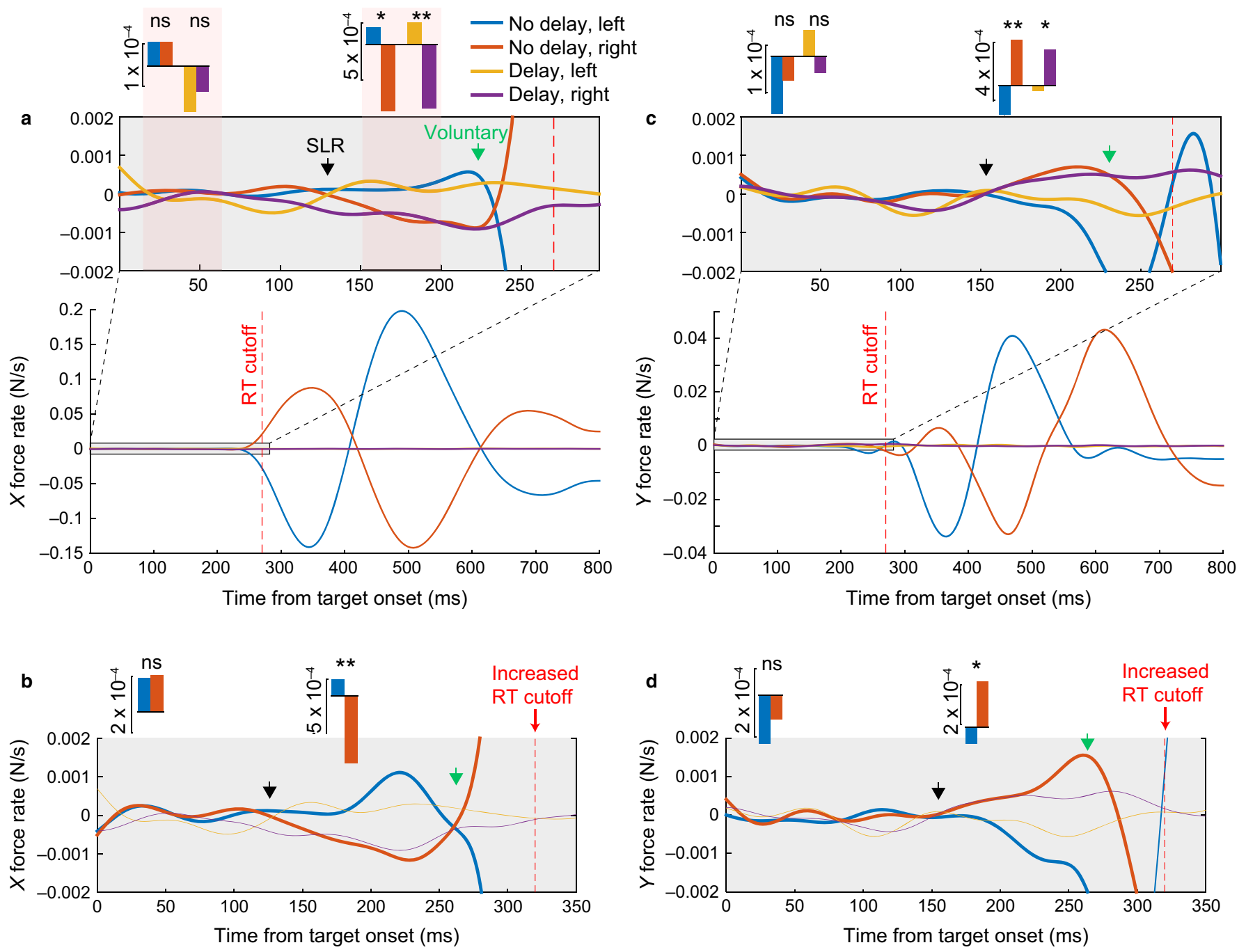

FIG. 6. The SLR influences manual forces. (a) Average force rates in the $x$-dimension during the instructed delay experiment. All trials with RTs below the cutoff (red dotted line: $270 \mathrm{~ms}$ ) were excluded. The inset plot with a gray background shows detail of the first $300 \mathrm{~ms}$ of the averages, revealing SLR-related separation at $\sim 150 \mathrm{~ms}$ (black arrow). Timing of voluntary forces is indicated by a green arrow. Red semi-transparent bands indicate SLR and baseline windows within which relationships between Target Left and Target Right conditions were statistically tested. (b) Detail of the first $350 \mathrm{~ms}$ for No Delay $x$-force rate averages after extending the RT cutoff by $50 \mathrm{~ms}$, showing that the timing of the separation between left and right target trials is tied to stimulus onset rather than voluntary RT. Delay averages (not affected by RT cutoff value) are shown for comparison. (c and d) Same as a and b, but for force rates in the $y$-dimension. $* P<0.05, * * P<0.01$.

gated whether this oppositely oriented bump could be attributable to an interaction torque. That is, if the shoulder/chest exerts a torque on the humerus in the direction of the target for the voluntary movement, it might cause the hand to briefly move in the opposite direction before the tricep/bicep compensates for that torque.

To address this, we extended the RT cutoff of the No Delay trials by $50 \mathrm{~ms}$ (i.e. we discarded RTs of $<320 \mathrm{~ms}$ ). This pushed back the average time of initiating voluntary forces by $50 \mathrm{~ms}$. If the separation of Target Left and Target Right conditions at $150 \mathrm{~ms}$ was merely an early stage of the voluntary movement forces (e.g. an interaction torque), we would expect to see that separation delayed by $50 \mathrm{~ms}$ also. If it was stimulus-locked, we would expect it to remain at $150 \mathrm{~ms}$. We observed the latter. The $x$-force and $y$-force rates of the two target direction conditions were significantly different during the $150-200-\mathrm{ms}$ window $(P=0.0027$ and $P=0.0274$, respectively), but not during the baseline window $(P=0.95$ and $P=0.54$, respectively). Moving the RT cutoff even further to $370 \mathrm{~ms}$ had no effect on these statistics or the timing of the SLR bump at $150 \mathrm{~ms}$. These analyses rule out the idea that the bump at $150 \mathrm{~ms}$ is related to voluntary movement. Instead, they suggest that the bump is stimulus-locked.

In our EMG recordings, the first 'rebound' of the SLR was often more pronounced than the initial wave of excitation or suppression, particularly in Target Right trials (Fig. 4). We suggest that the bump in force rate at $150 \mathrm{~ms}$ is the rebound of the SLR. This is supported by four observations: (i) the timing of the bump remained unchanged with respect to the voluntary RT; (ii) the bump also occurred in the Delay Stim-locked condition, where there was no voluntary movement; (iii) the direction of the bump was opposite to the direction of the target; and (iv) the relationship between target direction and SLR magnitude was the same for the forces and the EMG traces (i.e. strong rebound in the Target Right direction, and weaker SLR and rebound in the Target Left direction). Although kinematic evidence of the SLR has been observed in macaque neck muscle activity (Corneil et al., 2008; Chapman \& Corneil, 2011), our results are the first to demonstrate a behavioral correlate of the 
SLR in humans. A similar analysis of the luminance contrast experiment (reported in Fig. S3) supported this conclusion.

\section{Arrival of the SLR reduces variability in muscle activity}

Long RTs in the No Delay epoch revealed stimulus-locked low-frequency (10-15 Hz) oscillations following the SLR (Fig. 4c-e). Spectral analysis demonstrated that these low-frequency oscillations were present in chest muscle activity throughout the entire experiment (Fig. 7; see Fig. S4 for a supplemental wavelet analysis). The exact range of oscillation frequencies observed for both experiments was $10-14 \mathrm{~Hz}$. We assumed that, if the SLR was resetting the oscillation phase, the ITPC of $10-15-\mathrm{Hz}$ oscillations would be selectively increased around the time of the SLR (i.e. $~ 100 \mathrm{~ms}$ post-stimulus). Even though the phase locking of the oscillations is plainly visible in the multiple SLR bands of the intensity plots in Fig. 4, we validated this observation with spectral decomposition of the EMG signal. By comparing ERSP (Eqn 1) and ITPC (Eqn 2), it is possible to establish whether stimulus-induced phase resetting has occurred, and whether or not it can be attributed to increases in spectral power.

In all stimulus-locked conditions, we observed a selective low-frequency $(10-20 \mathrm{~Hz})$ increase in ITPC, but not in ERSP, starting at $\sim 100 \mathrm{~ms}$. To statistically assess this effect, we used $95 \%$ confidence intervals to compare inter-subject median ITPCs and ERSPs for a baseline (100-50 ms pre-stimulus) and SLR (100-150 ms post-stimulus) window (Fig. 8). Across all subjects (including those who showed no evidence of SLR), there was a separation between SLR and baseline median ITPC only in a low-frequency band $(\sim 10$ $20 \mathrm{~Hz})$. In the Delay Stim-locked conditions, where the EMG signal
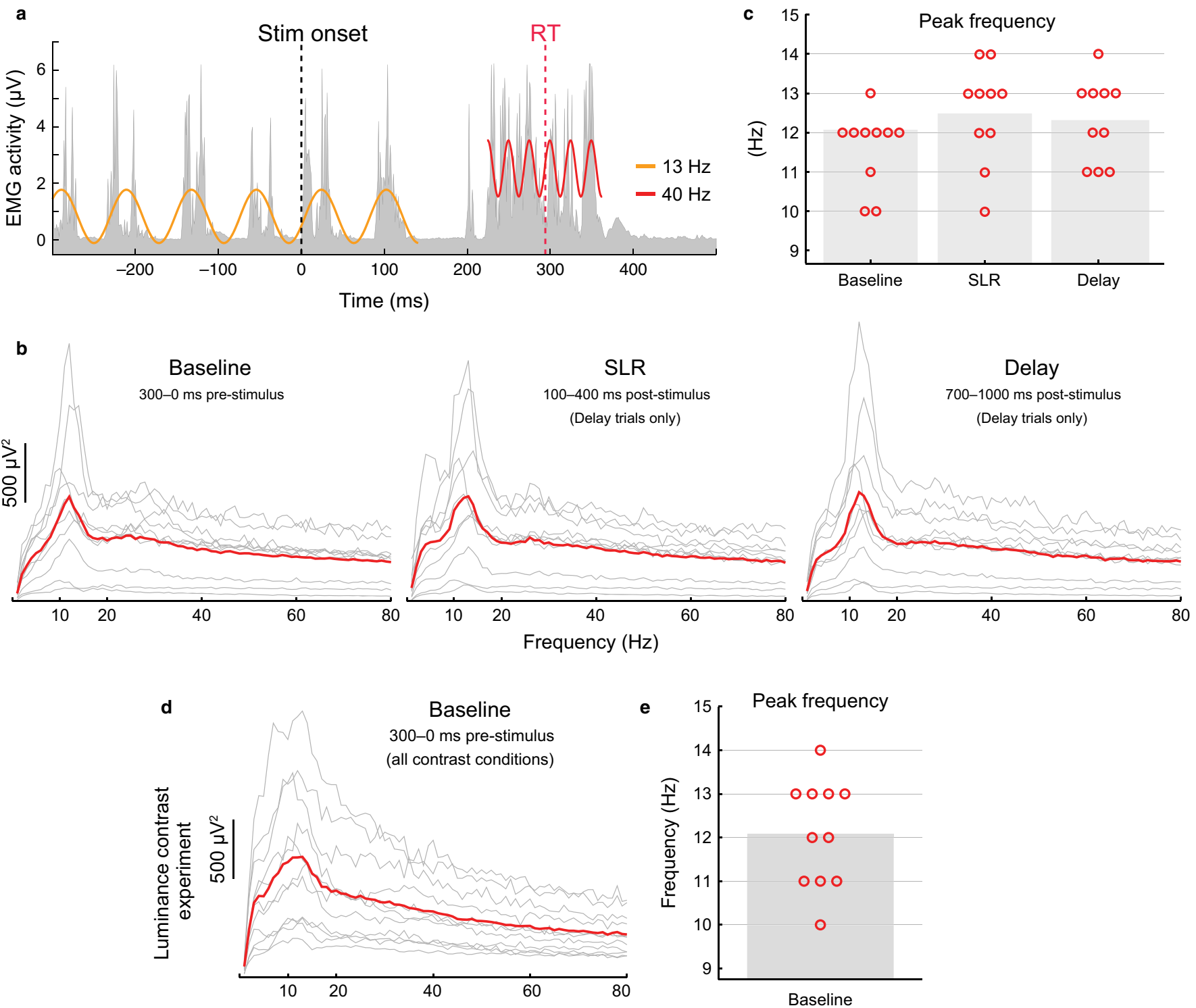

FIG. 7. Intrinsic oscillations in chest muscle EMG traces. (a) Oscillations in a single-trial EMG trace. A $13-\mathrm{Hz}$ oscillation dominates until the voluntary movement, where $40-\mathrm{Hz}$ oscillation dominates. (b) Single-sided power spectra for three different temporal windows: baseline, from 300 to 0 ms pre-stimulus; SLR, from 100 to $400 \mathrm{~ms}$ post-stimulus in the Delay condition (i.e. no reach after stimulus presentation); and delay, from 700 to 1000 ms post-stimulus in the Delay condition. The heavy trace is the between-subject average. (c) Frequency at peak power for each subject in each of the three epochs in b. (d and e) Oscillatory activity in the luminance contrast experiment. (d) Power spectrum for a baseline period in all contrast conditions. The heavy line is the average power across subjects. (e) Frequency at peak power for each subject. 

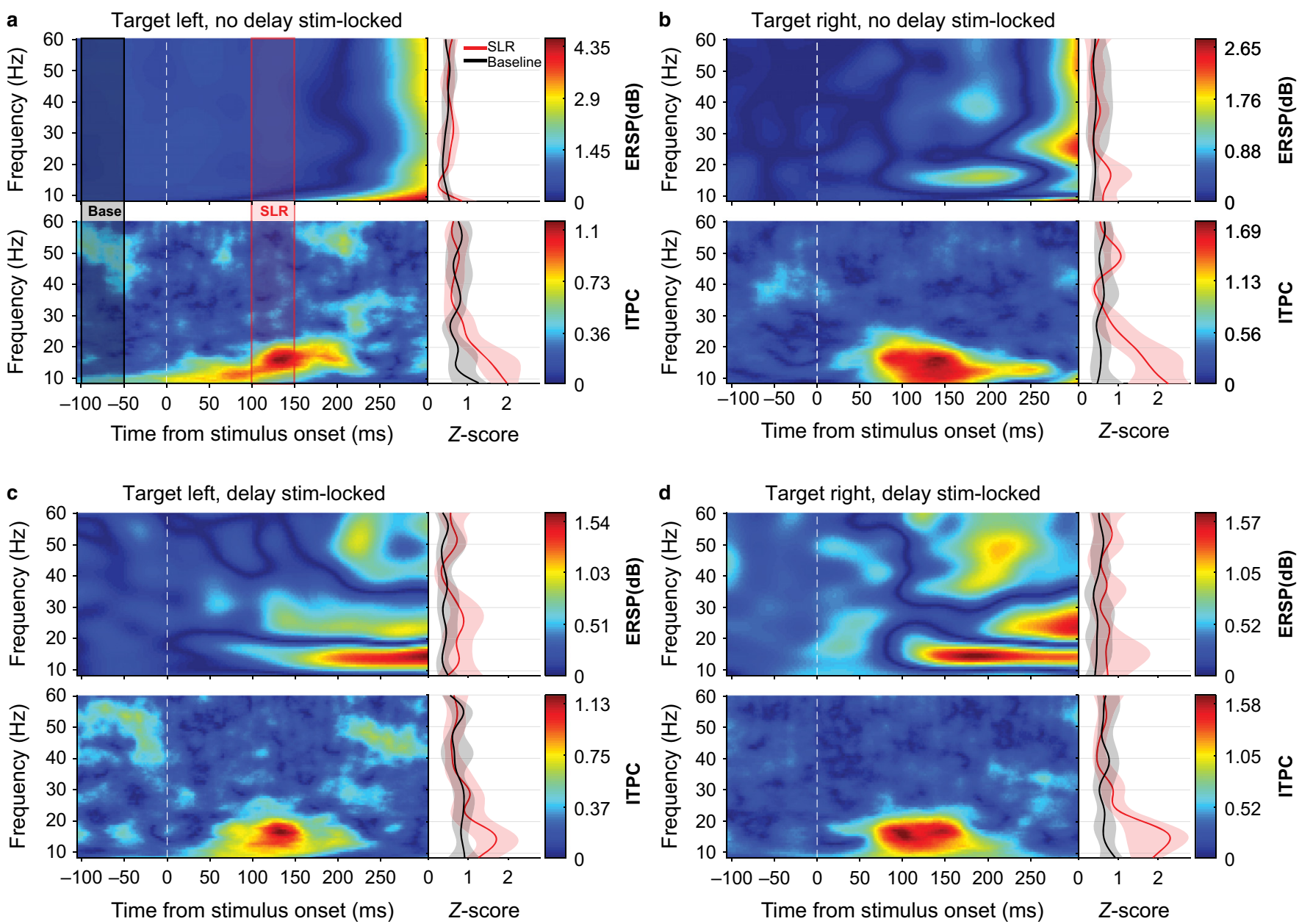

FIG. 8. SLR resets the phase of oscillatory activity. (a) Group-level normalised ERSP and ITPC for Target Left, No Delay Stim-locked trials. Line plots to the right of the time-frequency representations (TFRs) depict normalised median ERSP and ITPC \pm bootstrapped 95\% confidence intervals from a 50-ms baseline and SLR window (black and red transparent bars, respectively, over the TFRs). (b-d) Same as a, but for the other three Stim-locked conditions in the instructed delay experiment.

is less likely to contain very low frequency (i.e. $<5 \mathrm{~Hz}$ ) fluctuations attributable to the voluntary movement, there was a clear peak in the SLR-related ITPC increase at $\sim 15 \mathrm{~Hz}$. There were no differences between SLR and baseline ERSP, suggesting that the ITPC increases at the SLR were not attributable to event-related increases in spectral power. These effects were far more pronounced when we performed this analysis only on those subjects who showed prior evidence of the SLR. A supplementary analysis, in which we evaluated the instantaneous circular variance of phase between trials, agreed with this main analysis (Fig. S5). We conclude that the SLR results in a resetting of the phase of the $\sim 10-14-\mathrm{Hz}$ tremor oscillations in the EMG recordings. In other words, the arrival of the visual information in the SLR results in a reduction in the variability of muscle activity.

\section{Discussion}

We have demonstrated that the peripheral nervous system provides a readout of the transformation of visual inputs to motor outputs. Low-latency SLRs were sensitive to target contrast, they were spatially and temporally dissociable from voluntary orienting, and they were elicited by stimulus onsets and offsets. Furthermore, the visual transient reduced the variability of muscle activity by resetting the phase of intrinsic oscillations, similarly to what has been described throughout the central nervous system.

\section{Dissociation of stimulus detection and voluntary orienting in limb muscle activity}

SLRs in human limb muscles have been reported previously (Pruszynski et al., 2010), but key features of these responses were contrary to what would be expected of a visual transient; they failed to appear when a voluntary movement was suppressed, and when the go-cue was a stimulus offset. However, Pruszynski et al. used a blocked design, resulting in repetition of trial types. Their null results can be explained by another established feature of the visual transient: that it extinguishes with repetition (Boehnke et al., 2011). Here, we randomised the trial order, and found that an SLR inexorably followed (and was spatially tuned to) target onset or start-position offset, even when the voluntary reach was suppressed. This spatial and temporal dissociation between the SLR and voluntary orienting, aside from being a key feature of the visual transient response, is consistent with previous findings of SLRs in the neck muscles of non-human primates (Corneil et al., 2008; Chapman \& Corneil, 2011).

During the Delay Go-locked epoch, the Target Left and Target Right SLRs were synchronous rather than reciprocal. Synchronous 
SLRs in a single muscle acting as agonist or antagonist are consistent with co-contraction (or co-relaxation) of an antagonistic muscle pair (Fig. 5c), and although they are also consistent with a temporal lag for the Target Left (agonist) SLR, an interaction between movement direction and delay condition is improbable. Furthermore, even if co-contraction is assumed, it is unclear whether it should be interpreted as a spatially untuned SLR or a spatially tuned SLR towards an imperative stimulus that requires only the maintenance of the hand at its current position. Given overwhelming evidence that the SLR is spatially tuned in all other circumstances, we argue that cocontraction in the Delay Go-locked SLR is a spatially tuned response towards a centrally located imperative stimulus. This is further evidence that stimulus detection is dissociated from voluntary orienting in limb muscle activity.

\section{An orienting reflex in human upper limb muscles}

The visual transient observed here resembles the orienting reflex, which is a constellation of involuntary muscle responses that rapidly align the eyes, head and forelimbs towards a salient stimulus (Hess et al., 1946). It is associated with pursuit and prey-capture behaviors, and it has been well preserved throughout vertebrate evolution, as shown by its presence in fish (Preuss et al., 2014), frogs (King et al., 1999), rodents (Dean et al., 1986), and cats (Sokolov, 1963). It is mediated by a pathway from the superior colliculus (SC; optic tectum in non-mammals) to the reticular formation (Dean et al., 1989). In non-human primates, it is probably mediated by tectoreticular projections from intermediate and deep layers of the SC that carry arm movement control signals (Werner et al., 1997a,b; Stuphorn et al., 1999, 2000; Song et al., 2011; Philipp \& Hoffmann, 2014).

Limb control projections from the SC, such as SC cephalomotor projections (Gandhi \& Sparks, 2007), may lack the strong gating by reticular omnipause neurons that saccadic burst generator cells receive (Corneil \& Munoz, 2014). This is consistent with our observation of contrast sensitivity in the latency and magnitude of the SLR. As early as the retina, increased luminance contrast of visual inputs results in higher firing rates and lower, less variable transduction latencies (Shapley \& Victor, 1978). This effect is preserved throughout early visual processing areas in the form of a transient visual onset response (Boehnke \& Munoz, 2008; Pooresmaeili et al., 2010; Marino et al., 2012; Tanaka et al., 2012). A lack of strong gating in the tectoreticulospinal pathway would allow this contrastsensitive transient response to propagate directly onto the limb muscles. Our finding adds to the nascent literature demonstrating contrast-based effects on transient visual responses in the peripheral nervous system. For example, a recent report has shown that transient pupil responses are modulated by luminance contrast (Wang et al., 2014).

\section{Linking physiological tremor to sensorimotor integration}

We observed a resting $10-14-\mathrm{Hz}$ oscillation in upper limb muscles. This oscillation, also known as physiological tremor (typically 8$12 \mathrm{~Hz}$ ), is found in healthy populations (Schäfer, 1886). Physiological tremor appears to be the result of a combination of mechanical resonance (Vernooij et al., 2013), signaling lags in the stretch reflex loop (Lippold, 1970), and central oscillatory generators (Hallett, 1998). Phase resetting of pathological tremor and voluntary rhythmic movement have previously been induced by mechanical perturbation (Britton et al., 1992) and motor-evoked potential via transcranial magnetic stimulation (Pascual-Leone et al., 1994). Here, we report a novel demonstration of phase resetting of healthy physiological tremor by a visual stimulus.

The functional relationship between physiological tremor and the upper limb orienting reflex (e.g. resetting of tremor phase by the SLR) demonstrated here is not surprising, given the shared pathway of both mechanisms. The tectoreticulospinal orienting reflex pathway (for the upper limb) runs from SC to contralateral pontomedullary reticular formation (PMRF) cells (Sooksawate et al., 2013) that encode arm movements (Davidson et al., 2007; Hirschauer \& Buford, 2015) and the stimulus-locked adjustments in posture that compensate for such movements (Schepens et al., 2008). Many tectorecipient PMRF cells also receive convergent inputs from the caudal fastigial nucleus (Takahashi et al., 2014), which is a cerebellar output nucleus essential for the regulation of posture (Sprague \& Chambers, 1954). Critically, physiological tremor, which is associated with the maintenance of posture, originates in a network that includes the cerebellum and the PMRF (Williams \& Baker, 2009; Williams et al., 2010). In summary, the PMRF integrates lowlatency sensorimotor information from the SC with tremorogenesis and postural control signals from the cerebellum. This anatomical linkage (along with our discovery of a functional linkage) between the visual transient and physiological tremor suggests a role of the latter in sensorimotor integration. One possible role, given that tremor oscillations also appear in afferent (dorsal root ganglia) signals (Baker, 2006), is the facilitation of heightened accuracy in sensed limb position prior to movement.

The SLR reset the phase of the $10-14-\mathrm{Hz}$ oscillations, consistent with previous findings of sensory-induced phase resetting (Kazantsev et al., 2004; Reimer \& Hatsopoulos, 2010; Holtzman \& Jörntell, 2011; Romei et al., 2012; Jutras et al., 2013) and variability reduction (Cohen \& Maunsell, 2009; Churchland et al., 2010) throughout the central nervous system. But what is the functional significance of stimulus-induced phase resetting? We list three possibilities. First, it can serve as an extended marker of the exact timing of a sensory event (Wu et al., 2011). Second, a visual transient can enhance sensory responses by resetting the phase so that the subsequent detailed sensory information arrives in phase with the event-locked oscillation (Lakatos et al., 2007). Third, a phase reset can reduce the latency and temporal variability of information transfer, especially when the oscillation is at a low frequency (Llinás, 2009). Given these features, sensory-evoked phase resetting is an ideal mechanism for hastening a reflexive online correction to a perturbation or unexpected stimulus (Aoi et al., 2013; Ito, 2013; Llinás, 2013).

\section{The functional significance of the SLR}

In humans, involuntary corrective muscle responses can occur within $80-100 \mathrm{~ms}$ of a visual perturbation or a jump in target location (Saijo et al., 2005; Fautrelle et al., 2010; Perfiliev et al., 2010), and these fast corrections are dissociable from slower, voluntary corrections (Day \& Brown, 2001). The fast online correction is mediated by a spinal circuit that modulates ongoing corticospinal drive, and it is abolished by lesions in the reticulospinal tract (Alstermark et al., 1990; Pettersson et al., 1997; Pettersson \& Perfiliev, 2002). Given that our results implicate a tectoreticulospinal pathway for the SLR, a tempting hypothesis is that the SLR is a modulatory signal that has significant kinematic repercussions only when it is modifying ongoing corticospinal drive; it may represent the first step in a two-stage process of online reach trajectory modification (Gomi, 2008; Fautrelle \& Bonnetblanc, 2012). In contexts prior to voluntary reach initiation (e.g. in the present study), where the corticospinal drive is absent, this corrective signal may express itself as nothing 
more than a brief, stimulus-locked pulse of muscle activity. It is also possible that the SLR is a vestigial orienting reflex pathway that was more critical to survival for our evolutionary antecedents than it is for us.

\section{Supporting Information}

Additional supporting information can be found in the online version of this article:

Fig. S1. Alternative SLR latency analysis for the luminance contrast task.

Fig. S2. Error rates and reaction times for the instructed delay task. Fig. S3. Manual forces during the luminance contrast experiment.

Fig. S4. Wavelet analysis of instructed delay EMG data.

Fig. S5. Alternative analysis showing that the SLR resets the phase of oscillatory activity.

\section{Acknowledgements}

We thank Jakob Raum for assistance with data collection. We thank Jeremy Wong for assistance with the robot. We thank Haitao Yang for technical assistance. We also thank Andrew Pruszynski and Konrad Kording for helpful feedback on an earlier version of this manuscript. This work was supported by operating grants from the Natural Sciences and Engineering Research Council to M. A. Goodale (Grant 63132007 RGPIN), to P. L. Gribble (RGPIN-238338), and to B. D. Corneil (RGPIN-311680), and by grants from the Canadian Institutes of Health Research to D. K. Wood (Doctoral-CGS) and B. D. Corneil (MOP 93796).

\section{Abbreviations}

cPM, clavicular head of the right pectoralis major; EMG, electromyography; ERSP, event-related spectral perturbation; ITPC, inter-trial phase coherence; PMRF, pontomedullary reticular formation; ROC, receiver-operating characteristic; RT, reaction time; SC, superior colliculus; SEM, standard error of the mean; SLR, stimulus-locked response.

\section{References}

Alstermark, B., Gorska, T., Lundberg, A. \& Pettersson, L.G. (1990) Integration in descending motor pathways controlling the forelimb in the cat. 16. Visually guided switching of target-reaching. Exp. Brain Res., 80, 1-11.

Aoi, S., Kondo, T., Hayashi, N., Yanagihara, D., Aoki, S., Yamaura, H., Ogihara, N., Funato, T., Tomita, N., Senda, K. \& Tsuchiya, K. (2013) Contributions of phase resetting and interlimb coordination to the adaptive control of hindlimb obstacle avoidance during locomotion in rats: a simulation study. Biol. Cybern., 107, 201-216.

Baker, S.N. (2006) Afferent encoding of central oscillations in the monkey arm. J. Neurophysiol., 95, 3904-3910.

Boehnke, S.E. \& Munoz, D.P. (2008) On the importance of the transient visual response in the superior colliculus. Curr. Opin. Neurobiol., 18, 544-551.

Boehnke, S.E., Berg, D.J., Marino, R.A., Baldi, P.F., Itti, L. \& Munoz, D.P. (2011) Visual adaptation and novelty responses in the superior colliculus. Eur. J. Neurosci., 34, 766-779.

Britton, T.C., Thompson, P.D., Day, B.L., Rothwell, J.C., Findley, L.J. \& Marsden, C.D. (1992) 'Resetting' of postural tremors at the wrist with mechanical stretches in Parkinson's disease, essential tremor, and normal subjects mimicking tremor. Ann. Neurol., 31, 507-514.

Chapman, B.B. \& Corneil, B.D. (2011) Neuromuscular recruitment related to stimulus presentation and task instruction during the anti-saccade task. Eur. J. Neurosci., 33, 349-360.

Churchland, M.M., Yu, B.M., Ryu, S.I., Santhanam, G. \& Shenoy, K.V. (2006) Neural variability in premotor cortex provides a signature of motor preparation. J. Neurosci., 26, 3697-3712.

Churchland, M.M., Yu, B.M., Cunningham, J.P., Sugrue, L.P., Cohen, M.R., Corrado, G.S., Newsome, W.T., Clark, A.M., Hosseini, P., Scott, B.B.,
Bradley, D.C., Smith, M.A., Kohn, A., Movshon, J.A., Armstrong, K.M., Moore, T., Chang, S.W., Snyder, L.H., Lisberger, S.G., Priebe, N.J., Finn, I.M., Ferster, D., Ryu, S.I., Santhanam, G., Sahani, M. \& Shenoy, K.V. (2010) Stimulus onset quenches neural variability: a widespread cortical phenomenon. Nat. Neurosci., 13, 369-378.

Cohen, M.R. \& Maunsell, J.H.R. (2009) Attention improves performance primarily by reducing interneuronal correlations. Nat. Neurosci., 12, 1594 1600.

Corbetta, M. \& Shulman, G.L. (2002) Control of goal-directed and stimulusdriven attention in the brain. Nat. Rev. Neurosci., 3, 215-229.

Corneil, B.D. \& Munoz, D.P. (2014) Overt responses during covert orienting. Neuron, 82, 1230-1243.

Corneil, B.D., Olivier, E. \& Munoz, D.P. (2004) Visual responses on neck muscles reveal selective gating that prevents express saccades. Neuron, $\mathbf{4 2}$, 831-841.

Corneil, B.D., Munoz, D.P., Chapman, B.B., Admans, T. \& Cushing, S.L. (2008) Neuromuscular consequences of reflexive covert orienting. Nat. Neurosci., 11, 13-15.

Davidson, A.G., Schieber, M.H. \& Buford, J.A. (2007) Bilateral spike-triggered average effects in arm and shoulder muscles from the monkey pontomedullary reticular formation. J. Neurosci., 27, 8053-8058.

Day, B.L. \& Brown, P. (2001) Evidence for subcortical involvement in the visual control of human reaching. Brain, 124, 1832-1840.

Day, B.L. \& Lyon, I.N. (2000) Voluntary modification of automatic arm movements evoked by motion of a visual target. Exp. Brain Res., 130, $159-168$.

Dean, P., Redgrave, P., Sahibzada, N. \& Tsuji, K. (1986) Head and body movements produced by electrical stimulation of superior colliculus in rats: effects of interruption of crossed tectoreticulospinal pathway. Neuroscience, 19, 367-380.

Dean, P., Redgrave, P. \& Westby, G.W. (1989) Event or emergency? Two response systems in the mammalian superior colliculus. Trends Neurosci., 12, 137-147.

Delorme, A. \& Makeig, S. (2004) EEGLAB: an open source toolbox for analysis of single-trial EEG dynamics including independent component analysis. J. Neurosci. Meth., 134, 9-21.

Fautrelle, L. \& Bonnetblanc, F. (2012) On-line coordination in complex goal-directed movements: a matter of interactions between several loops. Brain Res. Bull., 89, 57-64.

Fautrelle, L., Prablanc, C., Berret, B., Ballay, Y. \& Bonnetblanc, F. (2010) Pointing to double-step visual stimuli from a standing position: very short latency (express) corrections are observed in upper and lower limbs and may not require cortical involvement. Neuroscience, 169, 697-705.

Fischer, B. \& Boch, R. (1983) Saccadic eye movements after extremely short reaction times in the monkey. Brain Res., 260, 21-26.

Gandhi, N.J. \& Sparks, D.L. (2007) Dissociation of eye and head components of gaze shifts by stimulation of the omnipause neuron region. $J$. Neurophysiol., 98, 360-373.

Gawne, T.J. (2002) Responses of primate visual cortical neurons to stimuli presented by flash, saccade, blink, and external darkening. J. Neurophysiol., 88, 2178-2186.

Gomi, H. (2008) Implicit online corrections of reaching movements. Curr. Opin. Neurobiol., 18, 558-564.

Gribble, P.L., Everling, S., Ford, K. \& Mattar, A. (2002) Hand-eye coordination for rapid pointing movements. Arm movement direction and distance are specified prior to saccade onset. Exp. Brain Res., 145, 372382.

Hallett, M. (1998) Overview of human tremor physiology. Movement Disord., 13, 43-48.

Henneman, E. (1957) Relation between size of neurons and their susceptibility to discharge. Science, 126, 1345-1347.

Hess, W.R., Bürgi, S. \& Bucher, V. (1946) Motor functions of tectal and tegmental areas. Monatsschr. Psychiatr. Neurol., 112, 1-52.

Hirschauer, T.J. \& Buford, J.A. (2015) Bilateral force transients in the upper limbs evoked by single-pulse microstimulation in the pontomedullary reticular formation. J. Neurophysiol., 113, 2592-2604.

Holtzman, T. \& Jörntell, H. (2011) Sensory coding by cerebellar mossy fibres through inhibition-driven phase resetting and synchronisation. PLoS One, 6, e26503.

Ito, M. (2013) Error detection and representation in the olivo-cerebellar system. Front. Neural Circuits, 7, 1.

Jutras, M.J., Fries, P. \& Buffalo, E.A. (2013) Oscillatory activity in the monkey hippocampus during visual exploration and memory formation. Proc. Natl. Acad. Sci. USA, 110, 13144-13149. 
Kazantsev, V.B., Nekorkin, V.I., Makarenko, V.I. \& Llinás, R.R. (2004) Self-referential phase reset based on inferior olive oscillator dynamics. Proc. Natl. Acad. Sci. USA, 101, 18183-18188.

King, J.G. Jr., Lettvin, J.Y. \& Gruberg, E.R. (1999) Selective, unilateral, reversible loss of behavioral responses to looming stimuli after injection of tetrodotoxin or cadmium chloride into the frog optic nerve. Brain Res., 841, 20-26.

Lakatos, P., Chen, C.-M., O’Connell, M.N., Mills, A. \& Schroeder, C.E. (2007) Neuronal oscillations and multisensory interaction in primary auditory cortex. Neuron, 53, 279-292.

Lakatos, P., Karmos, G., Mehta, A.D., Ulbert, I. \& Schroeder, C.E. (2008) Entrainment of neuronal oscillations as a mechanism of attentional selection. Science, 320, 110-113.

Lamme, V.A.F. \& Roelfsema, P.R. (2000) The distinct modes of vision offered by feedforward and recurrent processing. Trends Neurosci., 23, 571-579.

Lippold, O.C. (1970) Oscillation in the stretch reflex arc and the origin of the rhythmical, 8-12 C-S component of physiological tremor. J. Physiol., 206, 359-382.

Llinás, R.R. (2009) Inferior olive oscillation as the temporal basis for motricity and oscillatory reset as the basis for motor error correction. Neuroscience, 162, 797-804.

Llinás, R.R. (2013) The olivo-cerebellar system: a key to understanding the functional significance of intrinsic oscillatory brain properties. Front. Neural Circuits, 7, 96.

Marino, R.A., Levy, R., Boehnke, S., White, B.J., Itti, L. \& Munoz, D.P. (2012) Linking visual response properties in the superior colliculus to saccade behavior. Eur. J. Neurosci., 35, 1738-1752.

Nicolelis, M.A., Baccala, L.A., Lin, R.C. \& Chapin, J.K. (1995) Sensorimotor encoding by synchronous neural ensemble activity at multiple levels of the somatosensory system. Science, 268, 1353-1358.

Pascual-Leone, A., Valls-Solé, J., Toro, C., Wassermann, E.M. \& Hallett, M. (1994) Resetting of essential tremor and postural tremor in Parkinson's disease with transcranial magnetic stimulation. Muscle Nerve, 17, 800-807.

Perfiliev, S., Isa, T., Johnels, B., Steg, G. \& Wessberg, J. (2010) Reflexive limb selection and control of reach direction to moving targets in cats, monkeys, and humans. J. Neurophysiol., 104, 2423-2432.

Pettersson, L.G. \& Perfiliev, S. (2002) Descending pathways controlling visually guided updating of reaching in cats. Eur. J. Neurosci., 16, 13491360 .

Pettersson, L.G., Lundberg, A., Alstermark, B., Isa, T. \& Tantisira, B. (1997) Effect of spinal cord lesions on forelimb target-reaching and on visually guided switching of target-reaching in the cat. Neurosci. Res., 29, 241-256.

Philipp, R. \& Hoffmann, K.-P. (2014) Arm movements induced by electrical microstimulation in the superior colliculus of the macaque monkey. $J$. Neurosci., 34, 3350-3363.

Pooresmaeili, A., Poort, J., Thiele, A. \& Roelfsema, P.R. (2010) Separable codes for attention and luminance contrast in the primary visual cortex. $J$. Neurosci., 30, 12701-12711.

Preuss, S.J., Trivedi, C.A., vom Berg-Maurer, C.M., Ryu, S. \& Bollmann, J.H. (2014) Classification of object size in retinotectal microcircuits. Curr Biol., 24, 2376-2385.

Pruszynski, J.A., King, G.L., Boisse, L., Scott, S.H., Flanagan, J.R. \& Munoz, D.P. (2010) Stimulus-locked responses on human arm muscles reveal a rapid neural pathway linking visual input to arm motor output. Eur. J. Neurosci., 32, 1049-1057.

Purcell, B.A., Heitz, R.P., Cohen, J.Y. \& Schall, J.D. (2012) Response variability of frontal eye field neurons modulates with sensory input and saccade preparation but not visual search salience. J. Neurophysiol., 108, 2737-2750.

Reimer, J. \& Hatsopoulos, N.G. (2010) Periodicity and evoked responses in motor cortex. J. Neurosci., 30, 11506-11515.

Romei, V., Gross, J. \& Thut, G. (2012) Sounds reset rhythms of visual cortex and corresponding human visual perception. Curr. Biol., 22, 807-813.

Saijo, N., Murakami, I., Nishida, S. \& Gomi, H. (2005) Large-field visual motion directly induces an involuntary rapid manual following response. $J$. Neurosci., 25, 4941-4951.
Schäfer, E.A. (1886) On the rhythm of muscular response to volitional impulses in man. J. Physiol., 7, 111-117.

Schepens, B., Stapley, P. \& Drew, T. (2008) Neurons in the pontomedullary reticular formation signal posture and movement both as an integrated behavior and independently. J. Neurophysiol., 100, 2235-2253.

Schroeder, C.E. \& Lakatos, P. (2009) Low-frequency neuronal oscillations as instruments of sensory selection. Trends Neurosci., 32, 9-18.

Shapley, R.M. \& Victor, J.D. (1978) The effect of contrast on the transfer properties of cat retinal ganglion cells. J. Physiol., 285, 275-298.

Singh, K., Melis, E.H., Richmond, F.J.R. \& Scott, S.H. (2002) Morphometry of Macaca mulatta forelimb. II. Fiber-type composition in shoulder and elbow muscles. J. Morphol., 251, 323-332.

Sokolov, E.N. (1963) Higher nervous functions; the orienting reflex. Апnи. Rev. Physiol., 25, 545-580.

Song, J.-H., Rafal, R.D. \& McPeek, R.M. (2011) Deficits in reach target selection during inactivation of the midbrain superior colliculus. Proc. Natl. Acad. Sci. USA, 108, E1433-E1440.

Sooksawate, T., Isa, K., Matsui, R., Kato, S., Kinoshita, M., Kobayashi, K., Watanabe, D., Kobayashi, K. \& Isa, T. (2013) Viral vector-mediated selective and reversible blockade of the pathway for visual orienting in mice. Front. Neural Circuits, 7, 162.

Sprague, J.M. \& Chambers, W.W. (1954) Control of posture by reticular formation and cerebellum in the intact, anesthetized and unanesthetized and in the decerebrated cat. Am. J. Physiol., 176, 52-64.

Stuphorn, V., Hoffmann, K.-P. \& Miller, L.E. (1999) Correlation of primate superior colliculus and reticular formation discharge with proximal limb muscle activity. J. Neurophysiol., 81, 1978-1982.

Stuphorn, V., Bauswein, E. \& Hoffmann, K.-P. (2000) Neurons in the primate superior colliculus coding for arm movements in gaze-related coordinates. J. Neurophysiol., 83, 1283-1299.

Takahashi, M., Sugiuchi, Y. \& Shinoda, Y. (2014) Convergent synaptic inputs from the caudal fastigial nucleus and the superior colliculus onto pontine and pontomedullary reticulospinal neurons. J. Neurophysiol., 111, 849-867.

Tallon-Baudry, C., Bertrand, O., Delpuech, C. \& Pernier, J. (1996) Stimulus specificity of phase-locked and non-phase-locked $40 \mathrm{~Hz}$ visual responses in human. J. Neurosci., 16, 4240-4249.

Tanaka, T., Nishida, S., Aso, T. \& Ogawa, T. (2012) Visual response of neurons in the lateral intraparietal area and saccadic reaction time during a visual detection task. Eur. J. Neurosci., 37, 942-956.

Vernooij, C.A., Reynolds, R.F. \& Lakie, M. (2013) A dominant role for mechanical resonance in physiological finger tremor revealed by selective minimization of voluntary drive and movement. J. Neurophysiol., 109, 2317-2326.

Wang, C.A., Boehnke, S.E., Itti, L. \& Munoz, D.P. (2014) Transient pupil response is modulated by contrast-based saliency. J. Neurosci., 34, 408417.

Werner, W., Dannenberg, S. \& Hoffmann, K.-P. (1997a) Arm-movementrelated neurons in the primate superior colliculus and underlying reticular formation: comparison of neuronal activity with EMGs of muscles of the shoulder, arm and trunk during reaching. Exp. Brain Res., 115, 191-205.

Werner, W., Hoffmann, K.-P. \& Dannenberg, S. (1997b) Anatomical distribution of arm-movement-related neurons in the primate superior colliculus and underlying reticular formation in comparison with visual and saccadic cells. Exp. Brain Res., 115, 206-216.

Williams, E.R. \& Baker, S.N. (2009) Renshaw cell recurrent inhibition improves physiological tremor by reducing corticomuscular coupling at 10 Hz. J. Neurosci., 29, 6616-6624.

Williams, E.R., Soteropoulos, D.S. \& Baker, S.N. (2010) Spinal interneuron circuits reduce approximately $10-\mathrm{Hz}$ movement discontinuities by phase cancellation. Proc. Natl. Acad. Sci. USA, 107, 11098-11103.

Wood, D., Gallivan, J.P., Chapman, C.S., Milne, J.L., Culham, J.C. \& Goodale, M.A. (2011) Visual salience dominates early visuomotor competition in reaching behavior. J. Vision, 11, 16-16.

Wu, X., Ashe, J. \& Bushara, K.O. (2011) Role of olivocerebellar system in timing without awareness. Proc. Natl. Acad. Sci. USA, 108, 13818-13822. 